Michael Meyens Überblick über die Nutzungsgewohnheiten der Hörer in der DDR relativiert die Bedeutung bundesdeutscher Programmangebote. Denn fehlende Glaubwürdigkeit schloss offenbar die Nutzung nicht vordergründig aus. Die Attraktivität der Inlandssender beruhte auf den günstigen Empfangsbedingungen und vielfältigen Alltagsbezügen. Die Unterhaltungsorientierung des Publikums jedoch bedingte eine weit verbreitete Gleichgültigkeit gegenüber politischen Inhalten.

Auch wenn die Systematik der Anordnung der Beiträge nicht immer überzeugt, ist der Sammelband jedem Hörfunkinteressierten zu empfehlen. Die Beiträge beruhen zumeist auf der Auswertung archivarischer Quellen und behandeln überwiegend unberücksichtigte $\mathrm{Ge}$ genstände der Rundfunkgeschichte.

\section{Thomas Lietz}

\section{Allan Brown / Robert G. Picard (Hrsg.)}

\section{Digital Terrestrial Television in Europe}

Mahwah, New Jersey/London: Lawrence Erlbaum Assoc. Publishers, 2005.- 360 S.

ISBN 0-8058-5387-1

Nach der Digitalisierung der Produktion bemüht sich der Rundfunksektor seit einigen Jahren verstärkt um die Umstellung der Fernsehdistribution. Zwar soll diese Entwicklung möglichst marktgetrieben vonstatten gehen, im Kontext der Idee einer „europäischen Informationsgesellschaft" kommt der Implementierung der digitalen Übertragungstechnik - insbesondere in Bezug auf die Terrestrik - aber auch politische Bedeutung zu.

Vor diesem Hintergrund setzt sich der von Allan Brown und Robert G. Picard editierte Band aus medienökonomischer und -politischer Perspektive mit den europaweiten Entwicklungen im Bereich des digital-terrestrischen Fernsehens auseinander. Erstmals werden dabei ein ausführlicher Überblick über wesentliche Aspekte der Digitalisierung sowie detaillierte Darstellungen bisheriger Erfahrungen in ausgewählten europäischen Ländern geboten. Obwohl der Fokus auf dem terrestrischem Empfangsweg liegt, werden auch andere Formen des digitalen Fernsehens einbezogen. Hilfreich ist der Band weniger bei der Suche nach aktuellen Informationen - die Daten enden meist mit 2002, weshalb wesentliche Entwicklungen der letzten Jahre unberücksichtigt bleiben -, die Stärke liegt vielmehr im breit angelegten Zugriff und der fundierten analytischen Auseinandersetzung.

Der erste Teil beleuchtet in sechs Kapiteln länderübergreifend relevante Aspekte in Verbindung mit digital-terrestrischem Fernsehen. Fragen der Regulierung werden ebenso diskutiert wie Auswirkungen auf Fernsehinhalte, Konsumenten und die Rundfunklandschaft. Da mit Ausnahme des mobilen und portablen Fernsehens alle zentralen Gesichtspunkte behandelt werden, bieten die Beiträge einen vielseitigen Einblick in das Themengebiet.

Das Einführungskapitel widmet sich der kritischen Umstellungsphase von analoger auf digitale Übertragung. Nach Erörterung der Ausgangssituation sowie der technologischen Grundlagen wird näher auf die Herausforderungen für Rundfunkveranstalter, Netzbetreiber und Regulierung eingegangen. Medienpolitische Fragen der Digitalisierung auf EU-Ebene sind Thema des zweiten Kapitels. Mit Referenzen $\mathrm{zu}$ politischen und ökonomischen Entwicklungen zeichnet Pertti Näränen die europäische Position von den 80er Jahren bis 2002 nach; es geht dabei um länderübergreifende Frequenzplanung, die Etablierung von technischen Standards, die Regulierung der Märkte zur Verhinderung von Monopolstellungen sowie um Unterscheidungsprobleme von Rundfunk und Mediendiensten.

Herausforderungen der Digitalisierung für öffentlich-rechtliche und kommerzielle Rundfunkveranstalter werden in Kapitel drei und vier dargelegt. Im Vordergrund des Beitrags von Taisto Hujanen steht der Einfluss der zunehmenden Fragmentierung des Publikums auf die gesellschaftliche Bedeutung und politische Legitimation von Public-Service-Anbietern. Schon die Entstehung einer Viel-KanalUmgebung durch Kabel- und Satellitenfernsehen habe zu einem Wandel der Identität weg von der traditionellen Rolle als kulturelle Institution hin zu einem Selbstverständnis als konsumentenorientierter Programmanbieter geführt. Diese Tendenz werde durch die Digitalisierung noch verstärkt, wodurch das Konzept des öffentlich-rechtlichen Rundfunks zunehmend unter Legitimationsdruck gerate. Der anschließende Beitrag von Allan Brown befasst sich mit möglichen Konsequenzen für werbefinanzierte Rundfunkanbieter. Brown sieht in den erweiterten Sendekapazitäten des digitalterrestrischen Netzes wesentliches Verände- 
rungspotenzial. Einerseits werde dadurch die politische Kontrolle gelockert und durch Marktmechanismen ergänzt, was kommerziellen Anbietern den Zugang zu terrestrischen Frequenzen erleichtere. Andererseits könne die Vervielfachung des Angebots zu verstärkter Konkurrenz, sinkenden Werbeeinnahmen und steigenden Produktionskosten führen. Dennoch wird die Digitalisierung nach Ansicht des Autors die grundlegenden Strukturen der Medienlandschaft nicht verändern. Zusätzliche Fernsehanbieter könnten sich je nach regulatorischem Spielraum aber durchaus etablieren.

Als wesentliche inhaltliche Neuerung des digitalen Fernsehens werden gemeinhin interaktive Fernsehformate und Zusatzangebote gehandelt. Dieser Form des Fernsehens widmet sich das fünfte Kapitel. Nach dem Versuch einer Begriffsklärung und Typologisierung interaktiver TV-Dienste gibt Jens F. Jensen einen Überblick über die wichtigsten derzeit verfügbaren Fernsehinhalte dieses Bereichs. Genauer erklärt werden EPG (Electronic Programm Guide), Enhanced TV, Content-on-Demand, Personalised TV, Internet@TV, iTV Werbung, T-Commerce, Spiele, Wetten und Cross Media Interaction.

Am Ende des ersten Teils ergänzt Robert G. Picard die vorwiegend medienpolitische und -ökonomische Perspektive um den Blick auf die Konsumenten. Diskutiert werden weniger die Interessen zukünftiger Nutzer oder mögliche Veränderungen ihres Rezeptionsverhaltens, sondern in erster Linie der Aspekt der Nachfrage als kritischer Faktor für den Erfolg von iTV und DTV. Die Tatsache, dass die Digitalisierung zu Beginn stark von Regierungen und Medienunternehmen ohne Rücksichtnahme auf Konsumentenwünsche vorangetrieben wurde, sieht Picard als ernst zu nehmendes Hindernis für die Implementierung der neuen Technik.

Der zweite, deutlich umfangreichere Teil des Buches beleuchtete Prozesse, Erfahrungen und Resultate jener europäischen Länder, die zum Zeitpunkt des Redaktionsschlusses bereits mit der Einführung von digital-terrestrischem Fernsehen begonnen hatten oder kurz davor standen. Dabei handelt es sich um Großbritannien, Spanien, Schweden, Finnland, Dänemark, Frankreich, Deutschland und Italien. In den acht Fallstudien werden nicht nur Erfolge, sondern auch Schwierigkeiten und Fehlschläge der Frühphase von DTTV erörtert und auf Ursa- chen hin untersucht. Eine Fülle an Hintergrundinformationen ermöglicht dem Leser einen guten Einblick in Zusammenhänge zwischen strukturellen Rahmenbedingungen, Strategien von Medienunternehmen und politischen Entscheidungen der jeweiligen Länder. In der Zusammenschau der Beiträge zeichnen sich darüber hinaus auch allgemeine Mechanismen ab.

Abgeschlossen wird der Band mit einem Resümee der Herausgeber, das noch einmal alle thematischen Stränge zueinander in Verbindung setzt und mit Beispielen aus den Länderberichten untermalt. Zusammenfassend wird deutlich, dass die digitale Technologie der analogen zwar überlegen ist, die vollständige Digitalisierung der Fernsehdistribution für Medienpolitik, Rundfunkindustrie und Konsumenten aber nicht nur Vorteile, sondern auch Risiken, Unsicherheiten und Herausforderungen mit sich bringt.

\section{Christina Ortner}

\section{Werner Wirth / Edmund Lauf / Andreas Fahr (Hrsg.)}

\section{Forschungslogik und -design in der Kom- munikationswissenschaft}

Band 1: Einführung, Problematisierungen und Aspekte der Methodenlogik aus kommunikationswissenschaftlicher Perspektive

Köln: Halem, 2004. - 272 S.

ISBN 3-931606-53-8

Wie die Herausgeber des Buches in ihrer Einleitung zu Recht feststellen, gibt es keine eigenständige Methodologie, durch welche sich die Kommunikationswissenschaft von anderen empirischen Sozialwissenschaften unterscheiden würde. Damit stellt sich die Frage, worin der Sinn einer eigenständigen Publikation über Forschungslogik und -design in der Kommunikationswissenschaft bestehen könnte. Die Antwort der Herausgeber lautet: „Probleme der Forschungslogik [zeigen sich] aus kommunikationswissenschaftlicher Perspektive wenn schon nicht in völlig neuer Form, dann doch in einer spezifischen Gewichtung" (S. 7). Angezielt ist vor diesem Hintergrund eine Auseinandersetzung mit der „Anwendung von Forschungslogik in zentralen Forschungsfeldern der Wirkungs- und Rezeptionsforschung" (S. 8), wobei die „empirische Beweis- und Ar- 